병원 근로자의 직무스트레스와 소진의 관계에 미치는 펀 리더십과 근로자 놀이성의 조절 효과

박희태 ${ }^{1}$, 배성윤 ${ }^{2}$, 지재훈 ${ }^{3}$, 이기효 ${ }^{1}$

${ }^{1}$ 인제대학교 보건대학원, ${ }^{2}$ 인제대학교 경영학부 및 보건대학원, ${ }^{3}$ 인제대학교 병원전략경영연구소

\title{
Moderating Effect of Playfulness and Fun Leadership on the Relationship between Job Stress and Burnout among Hospital Employees
}

\author{
Hee-Tae Park ${ }^{1}$, Sung-Yoon Bae ${ }^{2} \neq$, Jae-Hoon $\mathrm{Ji}^{3}$, Key-Hyo Lee ${ }^{1}$ \\ ${ }^{1}$ Graduate School of Public Health, Inje University, ${ }^{2}$ School of Management and Graduate School of Public
}

Health, Inje University, ${ }^{3}$ Center for Health Care Strategic Management, Inje University

<국문요약>

본 연구는 병원 근로자들의 직무스트레스와 소진의 관계에 미치는 펀 리더십과 근로자 놀이성의 조절효 과를 규명함으로써 향후 병원조직의 효과적인 인적자원관리에 필요한 기초자료를 제공하고자 하였다. 연구 자료는 부산지역 2개 병원에 근무하는 근로자(의사 제외)를 대상으로 구조화된 자기기입식 설문지를 통하 여 수집하였으며 총 230 부의 설문지를 배부하여 이 중 207부의 설문을 회수하였다. 설문 문항에는 응답자 의 일반적 특성, 직무스트레스 특성, 펀 리더십, 근로자 놀이성, 소진 등을 측정하는 항목들이 포함되었다. 자료 분석을 위해 SPSS WIN 18.0을 이용하여 빈도분석, 신뢰도분석, 상관관계분석과 조절회귀분석 등을 수행하였다.

주요 결과를 요약하면 다음과 같다. 첫째, 직무스트레스와 소진의 관계에서 펀 리더십은 직접 효과는 물 론 조절효과도 유의하게 나타나지 않았다. 둘째, 직무스트레스와 소진의 관계에서 근로자 놀이성은 소진에 직접적인 효과를 주는 변수임과 동시에, 낮은 유의수준 $(\mathrm{p}=0.053)$ 에서지만 직무스트레스와의 상호작용을 통 해 소진을 감소시키는 조절효과가 있는 것으로 나타났다. 본 연구는 병원 근로자의 업무 수행과 관련해 비 교적 관심을 받지 못했던 펀 리더십이나 근로자 놀이성 등과 같은 성격적 특성이 소진을 감소시키는 유효 한 조절변수가 될 수 있음을 검증해 보았다는 점에 그 의의가 있으며, 다만 후속 연구를 통해 추가적인 검 증이 필요할 것으로 판단된다.

Key Words : Fun Leadership, Playfulness, Job Stress, Burnout, Hospital 


\section{INTRODUCTION}

Among the most complex organizations are hospitals, which is operated by employees with different specialties at various responsibilities ranging from physicians to nurses, pharmacists, technicians and administrative staff. While hospitals became more and more highly specialized as medical technologies have advanced over the several decades, not until recently has the importance of human resources and their mental health been addressed in Korean healthcare setting. But ironical is that today's management environment turns much different than previous decades, with ever-increasing needs for motivated individual talents and high-performing teams who should own effective provision of services and management of customer needs under those high-tech, complex hospital environment[1][2].

The current business of healthcare services, indeed, faces radical environment changes, both internal and external. While it must externally survive increasing competition, labor mobility, and even changes in social values, it is also required to deal with organizational challenges from increased informatization, needs of decentralization in authority structure and employee empowerment. Increased uncertainty of business environment due to technological advances and rapidly changing health polices makes it necessary that hospitals transform into the consistent learning organization, leading to more stressful work environment for organizational members.

Compared to employees in other industries, those of healthcare service industry are exposed to rather higher dose of job stress under the current organizational environment. Hospital employees have to routinely deal with complicated requests from patients and their families, and they are also required to effectively coordinate those requests and medical needs in order to prevent adverse events. In addition to the intrinsic nature of healthcare as negative services, burgeoning organizational challenges such as conflicts from dual line of authority with less developed management skills, burden of overwork, and increased risk of medical disputes have potential to contribute to higher job stress level among hospital employees than those in other industries[3].

Burnout, another important concept discussed in this paper, has been perceived as the irreversible consequence of job stress, and it has been reported to have a negative impact on hospital employees' physical and mental health at various levels[4]. Unlike the stress in general that can be recovered to normal condition, burnout might not be recovered due to the failure in adaptation mechanism[5].

Accumulated over the long period, serious job stress and burnout could have negative impacts on both individuals and their organizations. Not only do they affect employees' physical and mental health at the individual level, but they also indirectly cause productivity loss, reduction of job satisfaction and organizational commitment, and financial consequences to the organization resulted from higher absenteeism and staff turnover rate[3][6][7][8]. Therefore, the identification of factors causing job stress and 
burnout among hospital employees and the development of their effective management strategies will be necessary steps to improve the organization's competitive advantage. This intervention is particularly relevant to hospitals because the job stress and burnout among hospital employees, many of them being direct and indirect caregivers, may impact the quality of healthcare services rendered to their patients.

But, while most organizations' efforts were historically put on improving work environments other than reorganizing job itself or increasing intrinsic motivation of employees, relatively little attention has been paid to the personal characteristics of employees as players of enjoyable game and fun leadership holders.

It is interesting, however, that several researchers began to pay attention to the role of employees' playfulness and fun leadership behaviors in order to reduce job stress and burnout levels at work environment.

Some researchers believe that they can increase employees' intrinsic motivation to the work and the creative job performance by encouraging 'playful mind-set' by which employees can perceive their work as a kind of playing enjoyable game[9][10][11][12]. Other researchers report that they can maximize organizational effectiveness by facilitating 'fun leadership' through which management can make employees find more excitement and joyfulness in their work and instill them deep pride and feeling of strong affiliation to the organization[13].

Though until recently many studies have shown the moderating effects of self-efficacy, emotional intelligence and leadership on job stress and burnout, only a few studies have been focused on the effects of fun leadership and employees' playfulness, with very little research conducted in the hospital setting. Therefore, in this paper we tried to identify the effects of playfulness and fun leadership on the relationship between job stress and burnout among hospital employees, discussing the possible application of those concepts into the hospital setting.

\section{METHOD}

\section{Study Materials and Data Collection}

Empirical data has been collected through a survey using self-administered, structured questionnaires given to 230 employees (nurses, medical technicians and administrative staffs) at two hospitals with more than 250 beds in Busan, Korea. The survey was conducted from April 4th through 17th, 2011, to which a total of 207 employees responded.

\section{Study Model}

Given well established association between job stress and burnout, this study is intented to elucidate the moderating effects of playfulness and fun leadership in reducing job stress and burnout among hospital employees. 


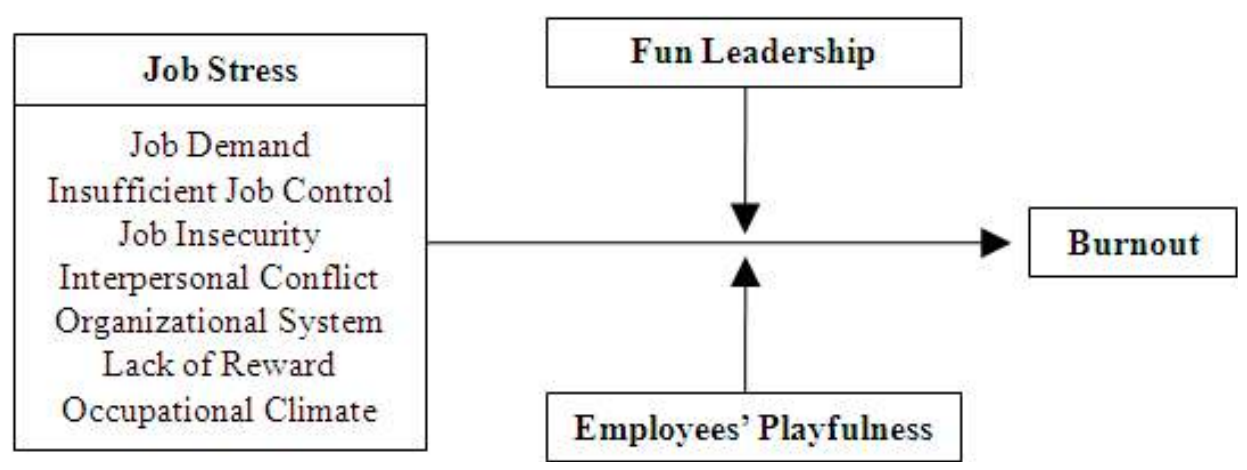

$<$ Figure $1>$ Study model

<Figure 1> depicts our study model, in which job stress as explanatory variable is speculated to influence on the employees' burnout level as response variable, and the association between two variables is possibly moderated by other elements such as employees' playfulness and supervisor's fun leadership behaviors. Further details on major instruments and other study questionnaires will be discussed in the following section.

\section{Instruments}

Structured questionnaire was used to measure major responses to the survey, and they consisted of 63 items in 5 parts, which included job stress, burnout, employees' playfulness, supervisor's fun leadership behaviors, and general characteristics of the survey respondents. Excluding those 8 items for general characteristics, most questions were presented in a five-point Likert scale, ranging from 1-point for 'strongly disagree' to 5-point for 'strongly agree.'
1) Job Stress

Questions for identifying job stress level consists of 24 items in 7 domains, which include job demand, insufficient job control, interpersonal conflict, job insecurity, organizational system, lack of reward, and occupational climate. They were measured using the short form of "Standardized Job Stress Measurement Scale for Korean Employees' developed by Chang et al.(2004)[14] and endorsed by Korean Society of Occupational Stress[15]. Reliability for the instrument has been tested with Cronbach's alpha value, which exceeded 0.5 in all domains(A instrument with Cronbach's alpha value of 0.5 or above is generally accepted as reliable for social science researches). Domain-specific Cronbach's alpha values were 0.703 for job demand, 0.594 for insufficient job control, 0.587 for interpersonal conflict, 0.570 for job insecurity, 0.721 for organizational system, 0.665 for lack of reward, and 0.583 for occupational climate, respectively<Table $1>$. 


\section{2) Employees' Playfulness}

Lieberman(1965) was among the first who defined playfulness as a characteristic of the player, and supported the existence of the playfulness trait in young children[16]. She defined playfulness as 'an internal predisposition to bring a playful quality to interactions and episodes.' This trait is an individual characteristic and its expression, relatively stable, reproducible and recognizable. In her study, Lieberman also identified five components of this playfulness quality: (a) physical spontaneity, (b) social spontaneity, (c) cognitive spontaneity, (d) manifest joy, and (e) sense of humor. The first instrument to measure playfulness in adults was developed by Glynn and Webster(1992)[17], but we used a modified version of Shaefer \& Greenberg's 'Playfulness Scale for Adults(PSA)' in our study[18][19]. Chronbach's alpha value for the instrument was 0.937, suggesting quite high level of its reliability<Table $1>$.

\section{3) Supervisor's Fun Leadership Behaviors}

Though recently getting its popularity among Korean management, 'fun leadership' is still unfamiliar to most researchers. Only recently has its conceptualization been attempted by Lee \& Chae(2008)[13]. In their study, Lee \& Chae defined fun leadership as "the leaders' coordinating skill or process to maximize organizational performance by encouraging employees to feel pride, fun and joyfulness to their job and the organization," and they reported through the review of previous literatures that supervisor's fun leadership behaviors increased job satisfaction as well as motivation and productivity while reducing job stress among organizational members. They also developed in their study the instrument to measure fun leadership, which is categorized into three components: smile, humor, and compliment. In our study, fun leadership behaviors was measured using a modified version of the scale developed by Lee \& Chae, focusing on the smile component, which reflects the direct expression of supervisor's emotional condition. Chronbach's alpha value for our version of the instrument was 0.860 , slightly lower than that of Lee \& Chae's original version $<$ Table $1>$.

\section{4) Burnout}

Burnout is the emotional condition of showing helplessness, frustration, and negative attitude to the self, others and occupation resulted from the consistent and repetitive emotional pressure related to job stress[20]. In our study, employees' burnout level was measured using a selected set of items from the 'Maslash Burnout Inventory(MBI)' developed by Maslach and Jackson(1981)[21]. We selected 5 items focusing on the emotional component of burnout from the instrument used by Kim \& Yoon(2008)[22]. Those selected items as the instrument measuring burnout turned out to be quite reliable with the Chronbach's alpha value of 0.838 , comparable to those of employees' playfulness and fun leadership<Table 1>. 
$<$ Table $1>$ Reliability of study measurement

\begin{tabular}{|c|c|c|c|}
\hline \multicolumn{2}{|c|}{ Variables } & \multirow{2}{*}{ The number of item } & \multirow{2}{*}{$\frac{\text { Cronbach's alpha }}{0.703}$} \\
\hline Job stress & Job demand & & \\
\hline & Insufficient job control & 4 & 0.594 \\
\hline & Interpersonal conflict & 3 & 0.587 \\
\hline & Job insecurity & 2 & 0.570 \\
\hline & Organizational system & 4 & 0.721 \\
\hline & Lack of reward & 3 & 0.665 \\
\hline & Occupational climate & 4 & 0.583 \\
\hline \multicolumn{2}{|l|}{ Playfulness } & 16 & 0.860 \\
\hline \multicolumn{2}{|c|}{ Fun Leadership } & 10 & 0.967 \\
\hline \multicolumn{2}{|l|}{ Burnout } & 5 & 0.838 \\
\hline
\end{tabular}

\section{Data Analysis}

SPSS 18.0 for Windows version was used for the analysis of data in this study. General characteristics of the survey respondents were presented in frequencies and percentages, and major variables such as job stress, burnout, employees' playfulness and their perception on supervisor's fun leadership behaviors were analyzed using t-test and ANOVA test to show their variations by general characteristics of the survey respondents. Reliability analysis was performed to test internal consistency of major instruments, and correlation analysis followed by moderated regression analysis was performed to examine the moderating effects of employees' playfulness and their supervisor's fun leadership on the relationship behaviors between job stress and burnout.

\section{RESULTS}

\section{General Characteristics of Survey Respondents}

General characteristics of a total of 207 survey respondents are presented in $<$ Table 2>. Among survey respondents was $86.5 \%$ of female employees, typical of human resources proportion found in Korean hospital setting. About two-thirds(70.5\%) were unmarried, and most of them( $89 \%)$ were under forties in their ages. Four-year college graduates or higher degree holders accounted for $38.6 \%$ of our survey respondents. About half(51.2\%) of employees have been working at their current hospital for more than two, but less than five years, while $38.6 \%$ worked for less than two years and $10.1 \%$ for more than five years. Nurses(69.1\%) were a majority of the respondents, while clinical technicians accounted for $14.5 \%$ and other administrative staffs for 
16.4\%. About two-thirds(62.8\%) worked split shifts in their hospital, and $69.1 \%$ of the survey respondents was paid less than 2 million Korean won for their monthly salary.

$<$ Table 2> General characteristics of survey respondents

\begin{tabular}{|c|c|c|c|}
\hline Variables & Category & Number & $\%$ \\
\hline \multirow{2}{*}{ Gender } & Male & 28 & 13.5 \\
\hline & Female & 179 & 86.5 \\
\hline \multirow{2}{*}{ Marital status } & Unmarried & 146 & 70.5 \\
\hline & Married & 61 & 29.5 \\
\hline \multirow{3}{*}{ Age(years) } & $20 \sim 29$ & 95 & 45.9 \\
\hline & $30 \sim 39$ & 89 & 43.0 \\
\hline & $40 \leq$ & 23 & 11.1 \\
\hline \multirow{2}{*}{ Education } & $\begin{array}{l}\text { diploma } \\
\text { holders or less }\end{array}$ & 127 & 61.4 \\
\hline & $\begin{array}{l}\text { 4-year college } \\
\text { graduates or higher }\end{array}$ & 80 & 38.6 \\
\hline \multirow{3}{*}{ Career(years) } & $<2$ & 80 & 38.6 \\
\hline & $2 \leq$ and $<5$ & 106 & 51.2 \\
\hline & 5 or more & 21 & 10.1 \\
\hline \multirow{3}{*}{ Job Category } & Nurse & 143 & 69.1 \\
\hline & Technician & 30 & 14.5 \\
\hline & $\begin{array}{l}\text { Administrative staff } \\
\ldots \ldots \ldots \ldots \ldots \ldots \ldots\end{array}$ & 34 & $\begin{array}{l}16.4 \\
\ldots . .\end{array}$ \\
\hline \multirow{2}{*}{ Work shift } & Yes & 130 & 62.8 \\
\hline & No & 77 & 37.2 \\
\hline \multirow{3}{*}{$\begin{array}{l}\text { Monthly pay } \\
\text { (KRW) }\end{array}$} & Less than 2 million & 143 & 69.1 \\
\hline & 2 million or more & 64 & 30.9 \\
\hline & Total & 207 & 100.0 \\
\hline
\end{tabular}

\section{Job Stress Level by General Characteristics}

The difference of domain-specific job stress level by general characteristics of our survey respondents is shown in <Table $3>$. Per job demand, job stress level was significantly different by marital status and work shift. Employees who were unmarried and worked in split shift felt more stress in their job. Statistically significant was the difference of job stress level in the domain of insufficient job control by marital status, age, length of career and monthly pay. Not surprisingly, married, less paid and younger employees who worked for less than two years turned out to have less control in their job, which contributed to higher job stress. Interpersonal conflict showed no statistically significant difference by general characteristics of the survey respondents.

Job stress caused by job insecurity was significantly different by the levels of education and monthly pay. Less educated, less paid employees felt more stress in their job. While occupational climate or organizational culture was of no statistical difference by general characteristics, organizational system was perceived differently among our survey respondents, in turn causing different level of job stress. In particular, employees in the age of twenties and clinical technicians felt more job stress caused by their negative perception on the organizational system. Lack of reward was reported among younger employees, especially those in the age of twenties, and it contributed more negatively to their job stress level. 
$<$ Table 3> Difference of job stress level by general characteristics

\begin{tabular}{|c|c|c|c|c|c|c|c|c|}
\hline \multirow{3}{*}{ Variable } & \multirow{3}{*}{ Category } & \multicolumn{7}{|c|}{ Job stress (7 domains) } \\
\hline & & Job demand & $\begin{array}{c}\text { Insuffi- } \\
\text { cient job } \\
\text { control }\end{array}$ & $\begin{array}{l}\text { Inter- } \\
\text { personal } \\
\text { conflict }\end{array}$ & $\begin{array}{c}\text { Job } \\
\text { insecurity }\end{array}$ & $\begin{array}{l}\text { Organi- } \\
\text { zational } \\
\text { system }\end{array}$ & $\begin{array}{l}\text { Lack of } \\
\text { reward }\end{array}$ & $\begin{array}{l}\text { Occu- } \\
\text { pational } \\
\text { climate }\end{array}$ \\
\hline & & M(SD) & M(SD) & M(SD) & M(SD) & M(SD) & M(SD) & M(SD) \\
\hline \multirow{3}{*}{ Gender } & Male & $3.24(.84)$ & 2.64(.65) & $2.68(.49)$ & 2.32(.76) & $3.16(.70)$ & $3.36(.71)$ & $2.55(.55)$ \\
\hline & Female & $3.34(.56)$ & 2.94(.79) & $2.37(.93)$ & 2.42(.64) & $3.15(.55)$ & $3.16(.66)$ & $2.65(.57)$ \\
\hline & $t(p)$ & $-.57(.57)$ & $-1.90(.06)$ & $1.68(.09)$ & $-.77(.44)$ & $.13(.89)$ & $1.46(.15)$ & $-.84(.40)$ \\
\hline \multirow{3}{*}{$\begin{array}{l}\text { Marit a I } \\
\text { status }\end{array}$} & Unmarried & $3.26(.62)$ & $3.26(.62)$ & $2.41(1.00)$ & $2.42(.66)$ & $3.18(.59)$ & $3.23(.66)$ & $2.65(.54)$ \\
\hline & Married & $3.46(.54)$ & $3.46(.54)$ & $2.44(.57)$ & 2.39(.66) & $3.08(.49)$ & $3.09(.67)$ & $2.61(.62)$ \\
\hline & $t(p)$ & $-2.18(.03)$ & $3.63(.00)$ & $-.23(.82)$ & $.36(.72)$ & $1.23(.22)$ & 1.32(.19) & $.43(.67)$ \\
\hline \multirow{4}{*}{$\begin{array}{l}\text { Age } \\
\text { (years) }\end{array}$} & $20 \sim 29$ & 3.34(.66) & $3.07(.74) b$ & $2.44(.58)$ & $2.41(.61)$ & $3.24(.61) b$ & $3.33(.61) b$ & $2.64(.54)$ \\
\hline & $30 \sim 39$ & $3.26(.56)$ & 2.83(.79)ab & $2.42(1.19)$ & $2.46(.68)$ & 3.10(.51)ab & $3.09(.65) a b$ & $2.65(.59)$ \\
\hline & $40 \leq$ & $3.48(.54)$ & $2.46(.64) a$ & 2.33(.63) & $2.26(.75)$ & $2.92(.55) \mathrm{a}$ & $2.99(.81) a$ & $2.59(.58)$ \\
\hline & $F(p)$ & $1.22(.30)$ & $6.84(.01)$ & $.12(.89)$ & $.81(.45)$ & $3.45(.03)$ & $4.21(.02)$ & $.10(.90)$ \\
\hline \multirow{3}{*}{ Education } & $\begin{array}{l}2-y r \text { diploma } \\
\text { holder or less }\end{array}$ & $3.31(.56)$ & $2.98(.77)$ & $2.37(.61)$ & $2.50(.69)$ & $3.17(.60)$ & $3.14(.71)$ & $2.67(.60)$ \\
\hline & $\begin{array}{l}4-y r \quad \text { college } \\
\text { graduate or higher }\end{array}$ & $3.30(.67)$ & 2.78(.78) & $2.49(1.21)$ & $2.26(.57)$ & $3.11(.52)$ & $3.27(.59)$ & $2.59(.51)$ \\
\hline & $t(p)$ & $-.22(0.83)$ & 1.86(.06) & $-.92(0.36)$ & 2.62(.01) & $.76(.45)$ & $-1.37(.17)$ & $.93(.36)$ \\
\hline \multirow{4}{*}{$\begin{array}{l}\text { Career } \\
\text { (years) }\end{array}$} & $<2$ & $3.32(.57)$ & $3.12(.73) b$ & $2.36(.57)$ & $2.48(.74)$ & 3.12(.63) & $3.25(.71)$ & 2.57(.62) \\
\hline & $2 \leq$ and $<5$ & 3.32(.65) & 2.79(.78)ab & $2.49(1.12)$ & $2.41(.58)$ & 3.19(.52) & $3.18(.65)$ & 2.72(.52) \\
\hline & 5 or more & $3.36(.53)$ & 2.62(.76)a & 2.22(.46) & 2.17(.62) & $3.04(.53)$ & $3.00(.52)$ & $2.48(.51)$ \\
\hline & $F(p)$ & $.04(.96)$ & 5.83(.01) & 1.04(.35) & $1.85(.16)$ & $.88(.42)$ & $1.20(.30)$ & $2.73(.07)$ \\
\hline \multirow{4}{*}{$\begin{array}{l}\text { Job } \\
\text { category }\end{array}$} & Nurse & $3.38(.51)$ & 2.93(.77) & 2.39(.98) & 2.44(.63) & 3.16(.53)ab & $3.16(.62)$ & $2.69(.56)$ \\
\hline & Technician & 3.12(.82) & $2.90(.72)$ & 2.38(.53) & $2.25(.58)$ & 3.38(.65)b & $3.39(.73)$ & $2.43(.46)$ \\
\hline & Admin. staff & $3.25(.70)$ & $2.76(.84)$ & $2.57(.73)$ & $2.44(.80)$ & $2.90(.56) a$ & $3.11(.78)$ & 2.58(.64) \\
\hline & $F(p)$ & $2.74(.07)$ & $.65(.53)$ & $.60(.55)$ & $1.05(.35)$ & 5.82(.01) & 1.73(.18) & $2.86(.06)$ \\
\hline \multirow{3}{*}{ Work shift } & Yes & $3.40(.51)$ & 2.95(.79) & $2.39(1.01)$ & $2.46(.65)$ & $3.18(.54)$ & $3.17(.63)$ & $2.69(.55)$ \\
\hline & No & $3.19(.72)$ & 2.82(.76) & $2.45(.64)$ & 2.33(.67) & 3.09(.60) & $3.21(.73)$ & $2.55(.57)$ \\
\hline & $t(p)$ & 2.34(.02) & $1.09(.28)$ & $-.49(.63)$ & 1.34(.18) & $1.04(.30)$ & $-.35(.73)$ & $1.80(.07)$ \\
\hline \multirow{3}{*}{$\begin{array}{l}\text { Monthly } \\
\text { pay } \\
\text { (KRW) }\end{array}$} & $<2$ million & $3.27(.63)$ & 2.98(.78) & $2.37(.60)$ & $2.47(.67)$ & $3.14(.60)$ & $3.14(.60)$ & 2.62(.54) \\
\hline & $\geq 2$ million & $3.44(.53)$ & $2.73(.75)$ & $2.53(1.33)$ & $2.27(.60)$ & $3.17(.48)$ & $3.17(.48)$ & 2.67(.61) \\
\hline & $t(p)$ & $-1.91(.06)$ & 2.18(.03) & $-1.19(.23)$ & 2.03(.04) & $-.38(.70)$ & $-.38(.70)$ & $-.60(.55)$ \\
\hline
\end{tabular}

\section{Burnout, Playfulness and Supervisor's Fun Leadership by General Characteristics}

The differences of burnout, employees' playfulness, and perception on supervisor's fun leadership behaviors by general characteristics are presented in $<$ Table $4>$. Employees' burnout level was significantly different by marital status, age and monthly pay. Higher level of burnout was found among unmarried, less paid, and 
younger employees in the age of twenties. Supposedly, a moderating factor in the relationship between job stress and burnout, employees' playfulness showed significant difference by marital status, education level, and monthly pay: unmarried, less educated and less paid employees had higher propensity to perceive their job as playing enjoyable game. As another potential moderator, employees' perception on their supervisor's fun leadership behaviors was significantly different by employees' monthly compensation level only: the less paid, the more likely do employees perceive their supervisors as fun leaders.

\section{Correlation of Major Variables}

Correlation among major variables of our study is presented in <Table 5>. Employees' burnout level as a response variable showed statistically significant correlation with all other explanatory variables in our study $(p=0.01)$, being negatively correlated with employees' playfulness and supervisor's fun leadership behaviors.

$<$ Table 4> Difference of burnout, playfulness and supervisor's fun leadership by general characteristics

\begin{tabular}{|c|c|c|c|c|}
\hline \multirow{2}{*}{ Variable } & \multirow{2}{*}{ Category } & Burnout & Playfulness & $\begin{array}{c}\text { Supervisor's fun } \\
\text { leadership }\end{array}$ \\
\hline & & M̈(SD) & $\mathrm{M}(\mathrm{SD})$ & $\mathrm{M}(\mathrm{SD})$ \\
\hline \multirow{3}{*}{ Gender } & Male & $2.89(.87)$ & $3.22(.52)$ & 3.06(.86) \\
\hline & Female & $2.84(.67)$ & $3.26(.48)$ & 3.27(.64) \\
\hline & $t(p)$ & $314(.754)$ & $-.459(.646)$ & $-1.516(.131)$ \\
\hline \multirow{3}{*}{$\begin{array}{l}\text { Marital } \\
\text { status }\end{array}$} & Ünmarried & $2.97(.72)$ & $3.31(.48)$ & $3.27(.67)$ \\
\hline & Married & $2.56(.52)$ & $3.13(.47)$ & $3.15(.70)$ \\
\hline & $t(p)$ & $4.479(.000)$ & $2.515(.013)$ & $1.164(.246)$ \\
\hline \multirow{4}{*}{$\begin{array}{l}\text { Age } \\
\text { (years) }\end{array}$} & $20 \sim 29$ & $3.07(.76) b$ & $3.29(.47)$ & $3.20(.60)$ \\
\hline & $30 \sim 39$ & 2.64(.59)a & 3.22(.52) & $3.30(.71)$ \\
\hline & $40 \leq$ & $2.73(.51) a b$ & $3.23(.40)$ & $3.12(.83)$ \\
\hline & $F(p) \quad \ldots \ldots \ldots \ldots \ldots \ldots \ldots \ldots \ldots \ldots \ldots \ldots \ldots \ldots$ & $10.133(.000)$ & $.514(.599)$ & $855(.427)$ \\
\hline \multirow{3}{*}{$\begin{array}{l}\text { Educatio } \\
n\end{array}$} & 2-yr diploma holder or less & $2.86(.71)$ & $3.35(.50)$ & $3.24(.75)$ \\
\hline & 4-yr college graduate or higher & $2.84(.67)$ & 3.11(.42) & $3.23(.55)$ \\
\hline & $t(p)$ & $.202(.840)$ & $3.691(.000)$ & $.121(.904)$ \\
\hline \multirow{4}{*}{$\begin{array}{l}\text { Career } \\
\text { (years) }\end{array}$} & $<2$ & $2.93(.66)$ & $3.32(.50)$ & $3.29(.69)$ \\
\hline & $2 \leq$ and $<5$ & $2.84(.71)$ & $3.22(.45)$ & $3.17(.67)$ \\
\hline & 5 or more & $2.60(.70)$ & $3.20(.56)$ & $3.35(.67)$ \\
\hline & $F(p)$ & 1.856(.159) & $1.235(.293)$ & $.999(.370)$ \\
\hline \multirow{4}{*}{$\begin{array}{l}\text { Job } \\
\text { category }\end{array}$} & Nurse & $2.89(.68)$ & $3.23(.48)$ & $3.21(.63)$ \\
\hline & Technician & $2.89(.84)$ & $3.29(.49)$ & $3.47(.66)$ \\
\hline & Administrative staff & $2.76(.64)$ & $3.32(.48)$ & $3.15(.86)$ \\
\hline & $F(p)$ & $306(.737)$ & $485(.617)$ & $2.258(.107)$ \\
\hline \multirow{3}{*}{$\begin{array}{l}\text { W o r k } \\
\text { shift }\end{array}$} & Yes & $2.85(.70)$ & $3.27(.49)$ & $3.25(.64)$ \\
\hline & No & $2.84(.70)$ & $3.24(.48)$ & $3.21(.75)$ \\
\hline & $t(p)$ & $.051(.960)$ & $.364(.717)$ & $437(.663)$ \\
\hline \multirow{3}{*}{$\begin{array}{l}\text { Monthly } \\
\text { pay } \\
(\mathrm{KRW})\end{array}$} & $<2$ million & $2.90(.74)$ & $3.32(.49)$ & $3.31(.70)$ \\
\hline & $\geq 2$ million & $2.71(.56)$ & 3.12(.43) & $3.07(.60)$ \\
\hline & $t(p)$ & 1.992(.048) & $2.774(.006)$ & $2.397(.017)$ \\
\hline
\end{tabular}


<Table 5> Correlation among major variables

\begin{tabular}{|c|c|c|c|c|c|c|c|c|c|}
\hline & Job demand & $\begin{array}{c}\text { Insufficient job } \\
\text { control }\end{array}$ & $\begin{array}{c}\text { Interpersonal } \\
\text { conflict }\end{array}$ & $\begin{array}{c}\text { Job } \\
\text { insecurity }\end{array}$ & $\begin{array}{c}\text { Organizational } \\
\text { system }\end{array}$ & $\begin{array}{l}\text { Lack of } \\
\text { reward }\end{array}$ & $\begin{array}{l}\text { Occupational } \\
\text { climate }\end{array}$ & $\begin{array}{c}\text { Fun } \\
\text { leadership }\end{array}$ & Playfulness \\
\hline $\begin{array}{l}\text { Insufficient job } \\
\text { control }\end{array}$ & 0.027 & & & & & & & & \\
\hline $\begin{array}{l}\text { Interpersonal } \\
\text { conflict }\end{array}$ & $0.181 * *$ & 0.104 & & & & & & & \\
\hline Job insecurity & $0.212 * *$ & 0.035 & $0.168 *$ & & & & & & \\
\hline $\begin{array}{l}\text { Organizational } \\
\text { system }\end{array}$ & $0.296 * *$ & $0.233 * *$ & $0.289 * *$ & $0.305 * *$ & & & & & \\
\hline Lack of reward & $0.230 * *$ & $0.182 * *$ & $0.293 * *$ & 0.113 & $0.574 * *$ & & & & \\
\hline $\begin{array}{l}\text { Occupational } \\
\text { climate }\end{array}$ & $0.258 * *$ & 0.103 & $0.341 * *$ & $0.343 * *$ & $0.440 * *$ & $0.491 * *$ & & & \\
\hline Fun leadership & $-0.251 * *$ & -0.121 & $-0.379 * *$ & $-0.241 * *$ & $-0.287 * *$ & $-0.376 * *$ & $-0.566 * \star$ & & \\
\hline Playfulness & $-0.193 * *$ & -0.055 & -0.117 & 0.100 & -0.740 & $-0.267 * *$ & -0.092 & $0.170 *$ & \\
\hline Burnout & $0.393 * *$ & $0.256 * *$ & $0.374 * *$ & $0.224 * *$ & $0.396 * *$ & $0.481 * *$ & $0.352 * *$ & $-0.329 * *$ & $-0.355 * \star$ \\
\hline
\end{tabular}

$\star p<.05, * * p<.01$

Per employees' playfulness, it was turned out to be negatively correlated with two domains of job stress, i.e. job demand and lack of $\operatorname{reward}(\mathrm{p}=0.01)$, while positively correlated with supervisor's fun leadership behaviors $(p=0.05)$. Employees' perception on their supervisor's fun leadership behaviors, however, was negatively correlated with all aspects of job stress except for job demand $(p=0.01)$.

\section{Effect of Playfulness on the Relationship between Job Stress and Burnout}

$<$ Table 6> shows the summary statistics of moderated regression analysis to examine the effect of employees' playfulness on the relationship between their job stress and burnout level. In regression model 1(the coefficient of determination, or $\mathrm{R}^{2}$ is equal to 0.357 ), where only one aggregated job stress variable as a sum of all seven domains was included, it was positively correlated with employees' burnout level.

Regression model 2 with additional explanatory variable, employees' playfulness, showed significant improvement in its statistical power $\left(\Delta \mathrm{R}^{2}=0.062\right)$, accounting for $41.9 \%$ of variation in the outcome variable, burnout $(p<0.001)$. In this model, employees' playfulness turned out to be negatively correlated with the burnout level.

However, in the regression model 3, where the interaction term of employees' playfulness and job stress was added to the regression model 2, further improvement of statistical $\operatorname{power}\left(\Delta \mathrm{R}^{2}=0.011, \Delta \mathrm{F}=3.785\right)$ has been observed at the marginal significance level of 0.053 , now accounting for $43.0 \%$ of variation in the burnout level. 
$<$ Table $6>$ Effect of playfulness on the relationship between job stress and burnout

\begin{tabular}{|c|c|c|c|c|c|c|c|c|c|c|c|c|}
\hline & \multicolumn{4}{|c|}{ Model 1} & \multicolumn{4}{|c|}{ Model 2} & \multicolumn{4}{|c|}{ Model 3} \\
\hline & B & beta & t-value & $p$ & B & beta & t-value & $p$ & B & beta & t-value & $p$ \\
\hline (Constant) & -.145 & & -.512 & .609 & 1.273 & & 3.127 & .002 & -2.381 & & -1.239 & .217 \\
\hline Job Stress & 1.031 & .598 & 10.676 & .000 & .950 & .551 & 10.143 & .000 & 2.183 & 1.266 & 3.408 & .001 \\
\hline Playfulness & & & & & -.363 & -.253 & -4.651 & .000 & .750 & .522 & 1.299 & .195 \\
\hline Job Stress $\times$ Playfulness & & & & & & & & & -.377 & -.957 & -1.945 & .053 \\
\hline$R^{2}$ & \multicolumn{4}{|c|}{.357} & \multicolumn{4}{|c|}{.419} & \multicolumn{4}{|c|}{.430} \\
\hline$\left(\Delta \mathrm{R}^{2}\right)$ & \multicolumn{4}{|c|}{.357} & \multicolumn{4}{|c|}{.062} & \multicolumn{4}{|c|}{.011} \\
\hline F-value change & \multicolumn{4}{|c|}{113.971} & \multicolumn{4}{|c|}{21.636} & \multicolumn{4}{|c|}{3.785} \\
\hline Sig. F-value change & \multicolumn{4}{|c|}{.000} & \multicolumn{4}{|c|}{.000} & \multicolumn{4}{|c|}{.053} \\
\hline
\end{tabular}

This result of moderated regression analysis suggests that employees' playfulness has a direct effect in relieving burnout among employees as well as a moderating effect of reducing the progression of job stress into burnout, albeit marginally significant $(p<0.1)$.

6. Effect of Perception on Supervisor's Fun Leadership Behaviors on the Relationship between Job Stress and Burnout

Presented in <Table $7>$ is the result of moderated regression analysis showing the effect of supervisor's fun leadership behaviors on the relationship between job stress and burnout level. Compared to the regression model $1\left(R^{2}=0.357\right)$, where only one aggregated job stress variable as a sum of all seven domains was included, regression model 2 with additional explanatory variable, employees' perception on their supervisor's fun leadership behaviors, showed little improvement in its statistical power.

$<$ Table $7>$ Effect of perception on supervisor's fun leadership behaviors on the relationship between job stress and burnout

\begin{tabular}{|c|c|c|c|c|c|c|c|c|c|c|c|c|}
\hline & \multicolumn{4}{|c|}{ Model 1} & \multicolumn{4}{|c|}{ Model 2} & \multicolumn{4}{|c|}{ Model 3} \\
\hline & B & beta & t-value & $p$ & B & beta & t-value & $p$ & B & beta & t-value & $p$ \\
\hline (Constant) & -.145 & & -.512 & .609 & -.105 & & -.214 & .831 & 1.078 & & .880 & .380 \\
\hline Job Stress & 1.031 & .598 & 10.676 & .000 & 1.025 & .594 & 8.896 & .000 & .642 & .372 & 1.688 & .093 \\
\hline Fun leadership & & & & & -.007 & -.007 & -.098 & .922 & -.361 & -.353 & -1.054 & .293 \\
\hline Job Stress $\times$ Fun leadership & & & & & & & & & .116 & .299 & 1.055 & .293 \\
\hline $\mathrm{R}^{2}$ & \multicolumn{4}{|c|}{.357} & \multicolumn{4}{|c|}{.357} & \multicolumn{4}{|c|}{.361} \\
\hline$\left(\Delta R^{2}\right)$ & \multicolumn{4}{|c|}{.357} & \multicolumn{4}{|c|}{.000} & \multicolumn{4}{|c|}{.004} \\
\hline F-value change & \multicolumn{4}{|c|}{113.971} & \multicolumn{4}{|c|}{.010} & \multicolumn{4}{|c|}{1.114} \\
\hline Sig. F-value change & \multicolumn{4}{|c|}{.000} & \multicolumn{4}{|c|}{.922} & \multicolumn{4}{|c|}{.293} \\
\hline
\end{tabular}


While the regression model 1 accounted for $35.7 \%$ of variation in the outcome variable, burnout $(p<0.001)$, the regression model 2 was not statistically significant.

However, even when the interaction term of supervisor's fun leadership behaviors and job stress variable as a sum of all seven domains was added, no significant improvement of the statistical power has been observed in the regression model 3, only accounting for $36.1 \%$ of variation in the burnout level.

The implication of this moderated regression analysis is that supervisor's fun leadership behaviors have neither a direct effect in alleviating employees' burnout level nor a moderating effect of hindering the progression of job stress into burnout.

\section{DISCUSSION \& CONCLUDING REMARKS}

This study was intended to examine job stress and burnout level among hospital employees, and the effect of employees' playfulness and their supervisor's fun leadership behaviors on the relationship between job stress and burnout, contributing to the development of effective strategies for the job stress management of human resources in the hospital setting.

Per job stress level among hospital employees, higher job demand, insufficient job control and lack of reward turned out to be the most important generators of job stress in our study. Higher level of job stress, particularly in job demand and job control areas, experienced among married employees in our study was not found in other studies, most of which focused on nurses[23][24]. This might have resulted from the higher sense of responsibility for their family with increased worries on their job skill and competence among married employees other than nurses. Consistent with results of other studies, stress from job insecurity was higher among less educated employees in our study, which is quite understandable considering the fact that the less educated, the less likely do they have sophisticated knowledge and skills, hence less capable of managing their stress.

Younger and less paid employees with careers of less than two years showed higher level of job stress, which is also consistent with results from other studies. Not surprisingly, those who work split shifts also showed higher level of job stress. This result of higher level of job stress experienced among younger, less paid workers, and those working split shifts suggests that measures to increase intrinsic motivation, employee empowerment and the sense of ownership are needed for the effective management of job stress among these groups of hospital employees.

However, the level of playfulness among hospital employees was higher in unmarried, less educated, and less paid group. This might be explained by the fact that married employees generally feel higher burden on their role and responsibility for their family, and that those with higher education are likely to have greater expectation to their job, taking their role rather seriously, hence less likely to enjoy their job.

Examined through moderated regression 
analysis, a statistically significant relationship was found among employees' playfulness, job stress and burnout. When job stress variable was included as the sole explanatory variable, the model accounted for only $35.7 \%$ of variation in the employees' burnout level(model 1). Adding another explanatory variable, employees' playfulness, increased statistical power of the model 1 by $6.2 \%$, enabling the model(model 2) to account for $41.9 \%$ of variation in the burnout level. However, when the interaction term of employees' playfulness and job stress variable was added to the model 2, further improvement of statistical power, albeit marginally, has been observed, and this model(model 3) was now able to account for $43.0 \%$ of all variations in the burnout level. This suggests that employees' playfulness is capable of directly relieving burnout, and at the same time it may reduce the progression of job stress into burnout through a moderating effect.

When the relationship of job stress, burnout and fun leadership was examined through moderated regression analysis, it was not statistically significant. To be specific, compared to the regression model 1 , where only job stress variable was included, the regression model 2 with additional explanatory variable, employees' perception on their supervisor's fun leadership behaviors, showed no improvement in its statistical power. Both regression models (model 1 and 2) accounted for $35.7 \%$ of variation in the outcome variable, burnout. Even when the interaction term of supervisor's fun leadership behaviors and job stress variable was added, significant improvement has not been observed in the statistical power (model 3). This result of moderated regression analysis suggests that supervisor's fun leadership behaviors have neither a direct effect in alleviating employees' burnout level nor a moderating effect of hindering the progression of job stress into burnout.

At our best available inference, the failure in identifying statistical correlation among job stress, burnout, and fun leadership behaviors could be attributed to the fact that we hired only a part of the instrument measuring fun leadership. As aforementioned, Lee \& Chae(2008)[13] developed the instrument to measure fun leadership, which consists of three components: smile, humor, and compliment. But we operationally defined the fun leadership by measuring the 'smile' component only in our study. This modification of the original instrument could have affected the result of moderated regression analysis, and therefore further study using a full version of the instrument will be helpful to verify the potential effect of fun leadership in reducing burnout level.

In this study, we tried to introduce the not-yet-fully-understood concepts of playfulness and fun leadership in the healthcare sector, analyzing their possible effects in reducing job stress and burnout level among hospital employees. Given the unfamiliarity of those concepts to most healthcare researchers, we would like to address on two challenges of our study to guide future researches on this subject.

First, the instruments we used to measure playfulness and fun leadership behaviors in our 
study might not be the best adapted tool for healthcare settings. To measure those concepts, we used modified versions of the questionnaires designed for general population or employees in general work setting, whose environment might be quite different from that of healthcare organizations. Most of current hospitals provide mainly negative services, which people are less likely to purchase voluntarily. Moreover, conventionally their organizational culture hardly allowed employees to pursue playfulness and fun behaviors at work. This suggests that different types of instrument to measure fun leadership might need in order to accommodate the uniqueness of hospital setting.

Second, we focused on mid-sized, secondary level hospitals only, and the results of our study might be limited in their generalizability to both primary and tertiary level healthcare organizations. Therefore, more studies need to be done in other healthcare organizations with different sizes in order to examine further applicability of the concepts covered in our study.

Despite the aforementioned challenges, we believe that our study has offered some important insights to both healthcare researchers and hospital managers, especially working in the organizational development area.

First, our study tried to specify the unfamiliar concepts of employees' playfulness and fun leadership behaviors still in a very nascent stage, successfully identifying empirical evidence on how those factors can affect job stress and burnout level among hospital employees.

Second, we believe that our study could be a reliable founding stone on which other researchers interested in this subject can explore further in the future. In real business world, however, hospital managers could develop strategies to more effectively deal with their employees' job stress and burnout level, hence improving organizational performance of their hospital.

\section{REFERENCES}

1. Park YJ(2009), "The Effect of Job Stress on Job Satisfaction and Turnover Intention among the Employee of Private Guard," Proceedings of 2009 Spring Conference, The Korea Contents Association, Vol.7(1);3-678.

2. Kang SM, Lee $\mathrm{KH}$, and Kim WJ(2010), "Influence of Leadership Perception of Hospital Employees on Organizational Efficacy and Controlling Effects of Individual and Organizational Characteristics," The Korean Journal of Health Service Management, Vol.4(2);47-61.

3. Koh JW, Suh YJ, and Park HY(1996), "The Effect of Job Stress and Social Support, on the Organizational Effectiveness of Hospital Employees," Journal of Preventive Medicine and Public Health, Vol.29(2);295-308.

4. Suh SR(1995), "Study on the Job Burnout of Special Education Teachers according to Internal Locus of Control and Social Support," The Graduate School of Ewha Womans University, pp.21-25.

5. Park MS(2004), "The Effects of Organizational Members' Job Burnout on Organizational Effectiveness," The Graduate School of Sogang University, pp.17-19. 
6. Beehr TA and Newman JE(1978), "Job Stress, Employee Health and Organizational Effectiveness; a Facet Analysis, Model and Literature Review," Personal psychology, Vol.31;665-669.

7. Gibson JL, Ivancevich JM, and Donnery IH(1985), "Organizations; Behavior Structure Process," 5th ed., Business Publ., p.223.

8. Moorhead G and Griffin RW(1992), "Organizational Behavior," Houshton-Miffin, 3rd ed., p.495.

9. Miller S(1973), "Ends, Means, and Galumphing: Some Leitmotifs of Play," American Anthropologist, Vol.75(1);87-98.

10. Starbuck WH and Webster J(1991), "When is Play Productive?," Accounting, Management and Information Technologies, Vol.1;71-90.

11. Webster J and Martocchio JJ(1992), "Microcomputer Playfulness: Development of a Measure with Workplace Implications," MIS Quarterly, Vol.16(2);201-226.

12. Webster J and Martocchio JJ(1993), "Turning Work into Play: Implications for Microcomputer Software Training," Journal of Management, Vol.19;127-146.

13. Lee YJ and Chae MS(2008), "An Empirical Study on the Influence of Fun Management and Fun Leadership Behavior on Job Stress and Job Attitude," Daehan Journal of Business, Vol.21(3);1029-1058.

14. Chang SJ, et al.(2004), "Study on the Development of Standardized Job Stress Measurement Scale for Korean Employees," The Second Yearly Report, The Korea Occupational Safety and Health Agency(KOSHA), p.171.

15. Korean Society of Occupational Stress, http://jobstress.or.kr, Accessed on August 10, 2011.

16. Lieberman JN(1965), "Playfulness and Divergent
Thinking: Investigation of Their Relationship at the Kindergarten Level," The Journal of Genetic Psychology, Vol.107;219-224.

17. Glynn MA and Webster J(1992), "The Adult Playfulness Scale: An Initial Assessment," Psychological Reports, Vol.71;83-103.

18. Shaefer $C$ and Greenberg R(1997), "Measurement of Playfulness: A Neglected Therapist Variable," International Journal of Play Therapy, Vol.2(6);21-31.

19. Yang ES(1998), "Relationship between Playfulness of the Play Therapists and a Career, an Attitude, Knowledge, and Skills," The Graduate School of Sookmyung Women's University, pp.62-64.

20. Yang JH and Jung $\mathrm{HY}(2009)$, "Relationship between Violence Response, Coping, and Burnout among Emergency Department Nurses," J. of Korean Acad Fundam Nurs, Vol.16(1);103-111.

21. Maslach C, and Jackson S(1981), "Maslach Burnout Inventory," Consulting Psychologist Press, Palo Alto, California, pp.2-40.

22. Kim YS and Yoon HS(2008), "A Study on Burnout and Organizational Effectiveness of Dental Hygienists," Journal of Dental Hygiene Science, Vol.8(4);291-297.

23. Kang HY(1992), "Study on the Relationship among Morale, Task Performance and Burnout Experiences of Nurses," Graduate School of Public Health, Chosun University, pp.30-34.

24. Kim EJ(2002), “The Relationship between Burnout Experiences and Job Satisfaction in Nurses," Graduate School of Public Administration, Kyung Hee University, pp.27-31.

접수일자 2011년 8월 16일 심사일자 2011년 9월 1일 게재확정일자 2011년 9월 13일 